\title{
Changes within bursts during learning in dissociated neural networks
}

\author{
Jan Stegenga, Joost le Feber, Wim L. C. Rutten
}

\begin{abstract}
We have studied the effect of imprinting a new stimulus-response (SR) relationship into a neuronal network cultured on a multi electrode array (MEA). We have used the Conditional Repetitive Stimulation (CRS) algorithm introduced by Shahaf et al in 2004. In this algorithm focal electrical stimulation is delivered at a low rate $(<1 \mathrm{~Hz})$ and is withdrawn when a desired response is observed. We confirmed that CRS could train the network to strengthen an initially weak SR relationship. With the acquisition of a new SR relationship, we studied its effect on network activity. Specifically, spontaneously occurring network bursts measured before, during and after training were analyzed. The total firing rate within bursts was estimated with a temporal resolution of milliseconds (burst profiles). We have shown earlier that these profiles change shape on a time base of several hours during spontaneous development. We show that the rate of change of the profiles during training (i.e. CRS) was higher than when no stimulation was applied.
\end{abstract}

\section{INTRODUCTION}

$\mathrm{T}$ HE processing of information in cultures of neuronal networks on Multi Electrode Arrays (MEAs) in absence of external stimuli is under the control of developmental processes. Alteration of processing by certain stimulation protocols (e.g. tetani) has been studied by many groups [15], with varying results. However, apart from inducing changes, where some activity-related measure changes as stimulation is applied, we ultimately want to be able to predict these changes and control them.

The Conditional Repetitive Stimulation (CRS) training protocol has been introduced by Shahaf et al [6]. It was successful in strengthening one particular electrode's response to a stimulus at another electrode. This kind of control has not been reported by other algorithms that are applicable to dissociated cultures. The feedback of the cultures' performance consisted of the withdrawal of the stimulus when the desired response was observed. The experiments were self-contained in that no further analysis of activity was needed to prove that a change in the network processing had occurred. Thus, apart from a series of test stimuli before and after the experiment, Shahaf et al did not report on additional analyses of (changes in) network activity.

We investigated the changes that occurred within the network in order to accommodate the desired stimulus-

Manuscript received April 16, 2008.

J. Stegenga, J le Feber and W. L. C. Rutten are all with the Biomedical signals and systems group (BSS), Institute for Biomedical Technology (BMTI), University of Twente, Enschede, The Netherlands. response (SR) relationship. Our approach describes the relationship of each electrode to network-wide synchronous events; bursts.

We previously analyzed the natural development of bursts in vitro through profiles of the instantaneous firing rate during bursts [7]. This revealed electrode specific contributions to bursts, which changed shape with a time-base of several hours. This method allowed us to study the progress of bursts during CRS training.

\section{METHODS}

\section{A. Culturing and Maintenance}

Cortices were taken from newborn Wistar rats. The cells were dissociated mechanically by trituration and chemically by trypsin. The centers of multi electrode arrays (MEAs) were coated with poly-ethyl-imine (PEI), after which a drop of medium was applied. The plating concentration was 1 million cells per $\mathrm{ml}$, which resulted in a monolayer of cells with a density of $\sim 2500$ cells per $\mathrm{mm}^{2}$ after 2 days in vitro (DIV). Cultures were stored in an incubator with $5 \% \mathrm{CO}_{2}$ to air mixture, and near $100 \%$ humidity at $37^{\circ} \mathrm{C}$. Cultures were refreshed twice a week with R12 culturing medium [8].

\section{B. Experimental setup}

We used MEAs and measurement setup (1060BC preamplifier and STG1002 stimulus generator) manufactured by MultiChannel Systems, Reutlingen, Germany. The MEAs had 60 electrodes which were either 10 or 30 um in diameter, which were spaced either 100 or 200 um apart, respectively. During measurements the temperature at the bottom of the MEA was controlled at $36^{\circ} \mathrm{C}$, and a humidified and heated stream of air with $5 \%$ $\mathrm{CO}_{2}$ was blown over the setup. Cultures were sealed with a semi-permeable membrane.

Measurements were controlled entirely by custom LabView (National Instruments, Austin, TX) programs. Spikes were detected whenever the signal crossed a threshold of 6 times the noise level, and were validated online using a scheme adopted from Wagenaar et al [9]. No spike sorting was applied.

\section{Conditional Repetitive Stimulation}

The Conditional Repetitive Stimulation (CRS) algorithm was introduced by Shahaf et al [6]. It was their observation 
that stimuli applied at a low rate $(0.3-1 \mathrm{~Hz}$, such that burst could be triggered) to a single electrode caused changes in functional connectivity within the network. The responses to the stimuli were monitored and when a desired response appeared often enough (responsiveness), the stimuli were stopped. A single response was defined as one or more spikes elicited by a single evaluation electrode within a certain window after stimulus onset (e.g. from 40-60 ms). The responsiveness was calculated as the moving average of the last ten responses, and the desired threshold was set at two out of ten, where the initial responsiveness was about one out of ten. When the desired condition for the responsiveness was met, or after 10 minutes of trying, the stimuli were withdrawn. For the training to be successful, the number of stimuli required to achieve the desired responsiveness when the stimuli were started again 5 minutes later, should be reduced.

Our procedure was as follows. After probing each electrode with stimuli of varying amplitude (4-20 uA, biphasic, $200 \mathrm{us} /$ phase, negative phase first), we selected an electrode which was able to trigger bursts with high reliability. Next, the responsiveness of each electrode was determined with a series of test stimuli delivered to the selected electrode. At this point, we also determined the frequency at which to stimulate such that bursts were triggered with maximum probability, while minimizing the number of spontaneous bursts in between. Therefore, inter stimulus intervals (ISI) varied between 1.5 and 5 seconds.

The evaluation electrode and the evaluation window were chosen such that 1) the responsiveness was about 0.1 and 2) the window corresponds to a peak in the post stimulus time histogram (PSTH) of the evaluation electrode. A varying window was preferred because the bursts varied by culture and by age, and because we wanted to change connection strength rather than latency. A learning experiment was stopped when a stable fast responsiveness was reached, or when the network wide response (i.e total number of spikes within $300 \mathrm{~ms}$ after stimulus) to stimuli became lower than $80 \%$ of its initial value. This initial value was calculated by taking the average of the first 5 iterations of the CRS algorithm. Every CRS experiment was preceded and followed by a measurement of spontaneous activity.

\section{Profiles}

We made profiles of the within burst firing rate. The procedure has been described earlier [7]. Shortly, bursts were detected by dividing the summed activity in $100 \mathrm{~ms}$ bins and applying a threshold. The threshold was set at 2 times the number of electrodes that showed activity. Next, a burst profile (BP) was calculated by convolving the spike times (summed over all electrodes) in a burst with a Gaussian. The standard deviations used were between 5 and $15 \mathrm{~ms}$, depending on activity. The width of the profiles was $600 \mathrm{~ms}$, and 601 samples were used. We used the root- mean-square (RMS) value of the difference between two profiles to quantify change. We normalized the RMS value to the RMS of the profile (i.e. the number of spikes):

$$
D_{i}=\frac{R M S\left(p_{i}-p_{\text {ref }}\right)}{R M S\left(p_{\text {ref }}\right)}, \operatorname{RMS}(x)=\operatorname{sqrt}\left(\frac{1}{N} \sum_{n=1}^{N} x_{n}^{2}\right)
$$

Where $D_{i}$ is the normalized distance between profile $p_{i}$ and a reference profile $p_{\text {ref }}$ and each profile consisted of $N=601$ samples. This distance measure is sensitive to changes in profile shape and to increases in firing rate. The rate of change of $D$ during experiments was calculated using the 5 minutes of spontaneous activity between iterations. The reference profile that we used within CRS is the average profile during the first 5 minute break between iterations. For spontaneous measurements we used the average profile during the first 5 minutes as a reference.

\section{RESULTS}

\section{A. CRS training}

The average learning curve of 12 experiments performed on 9 different cultures is shown in figure 1A. A decrease in the number of stimuli required to elicit the desired response could be seen. Individual curves, such as the examples shown in figure $1 \mathrm{~B}$ show that learning could be highly erratic. A period in which responsiveness was below initial responsiveness was not uncommon. The selectivity of CRS training is shown in figure $1 \mathrm{C}$ and $1 \mathrm{D}$, where the network response is compared with the response of the evaluation activity. It is clear that the increase in number of action
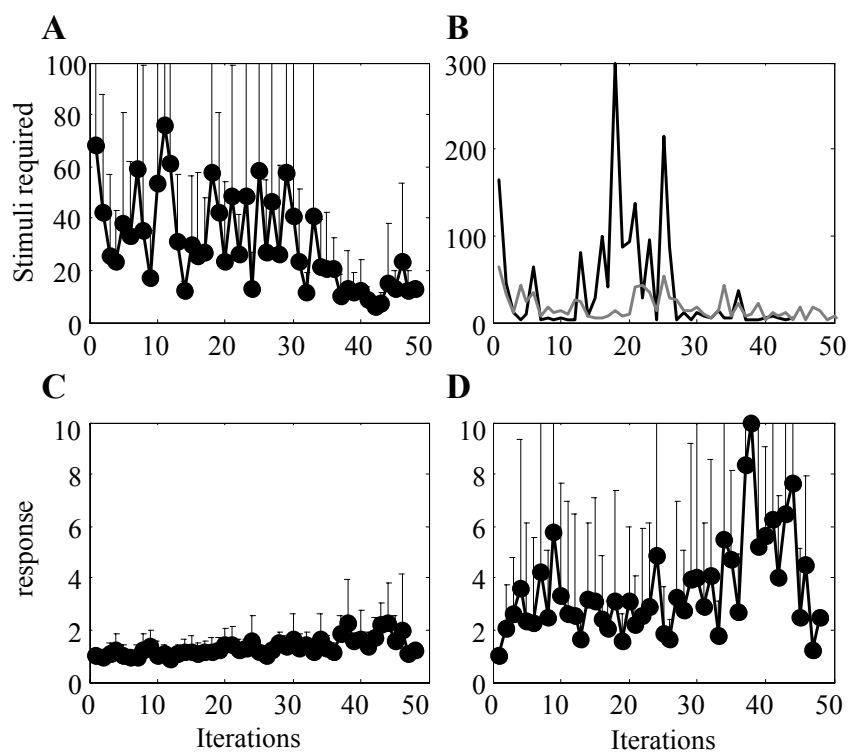

D

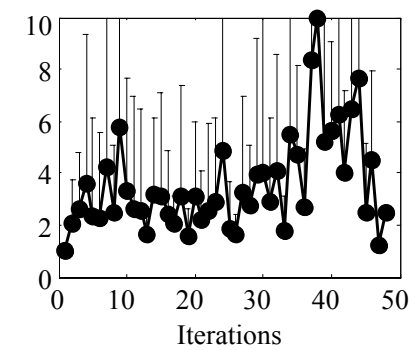

Fig. 1. Performance of the CRS training algorithm. A The average learning curve. B Examples of two individual learning curves show the erratic nature of the training process. C Network wide response, calculated as the total number of spikes elicited in the response window. The curve is normalized to the average value found in the first iteration. D Evaluated electrode response, calculated as the number of spikes on the evaluation electrode within the response window and normalized to the value found in the first iteration. Curves show means plus standard deviation. 

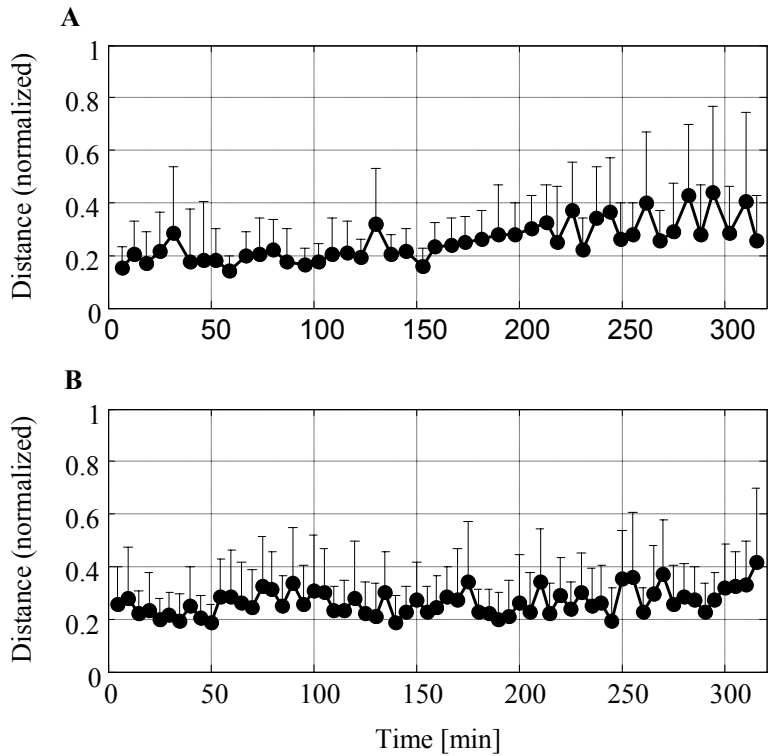

Fig. 2. Distances between burst profiles (BP). A Distances between burst profiles during CRS training. The average BP during the first iteration was used as reference. B Distances between burst profiles during spontaneous activity. The average BP during the first 5 minutes of recording served as a reference. Figure show means plus standard deviations.

potentials elicited within the evaluation window on the evaluation electrode is larger than the overall increase.

\section{B. Profiles}

The distances between burst profiles during CRS and during spontaneous measurements (taken immediately before or after CRS) are shown in figure 2. A lower bound of about 0.2 may represent the stochastic variability in bursts. A slope in the graph indicates that bursts change shape incrementally. It is clear that during CRS training, the bursts changed faster (higher slope) than during spontaneous measurements.

Figure 3 shows the burst profiles (left panels) and the contribution of evaluated electrodes to the burst profiles (right panels). The direction of change of local profiles on evaluated electrodes was not uniform. A decrease in peak firing rate was observed in most cases, but also shifts in the position of the main peak and in some cases an increasing second phase were observed. We have observed such changes during spontaneous activity as well, albeit that it is rare to observe an increase in second phase spontaneously.

\section{CONCLUSIONS}

We found it necessary to apply CRS for quite long (>30 iterations) to reach a stable low value, as initial learning was very erratic. Shahaf et al [6] observed a much faster learning rate in their experiments, often converging to a stable low value within 10-20 iterations. We also observed that, after we had selected an evaluation electrode and measured spontaneous activity, the criterion was reached very quickly
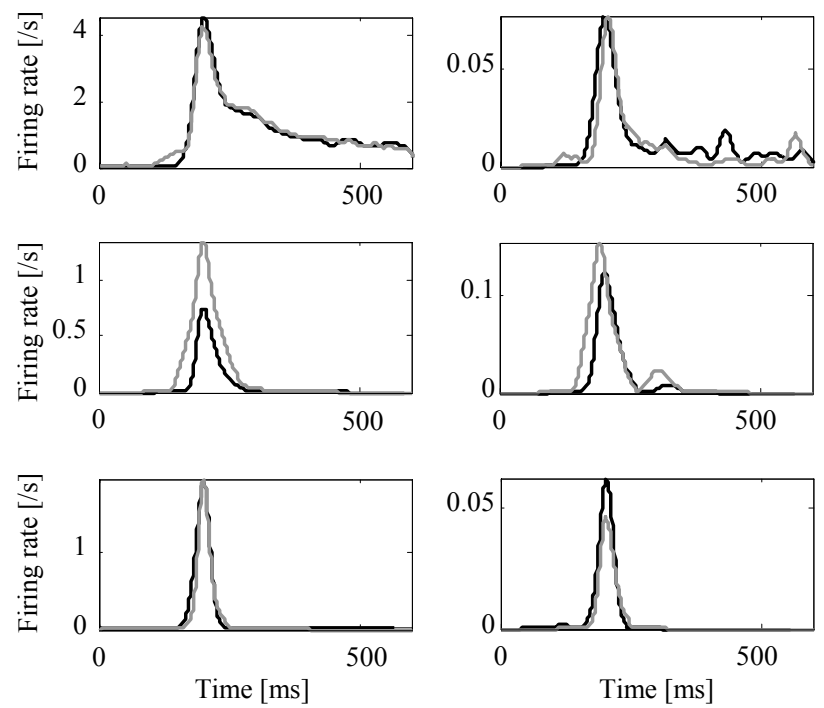

Fig. 3. Examples of burst profiles and contributions by evaluated electrodes. Left panels) Burst profiles at the start (black) and at the end (gray) of CRS training. Burst profiles are aligned o their peak at $200 \mathrm{~ms}$. Right panels) Phase profiles of evaluated electrodes at the start (black) and at the end (gray) of CRS training. Shifts in peakpostion, changes in peak firing rate and the acquisition of adstinct second phase were observed.

already at the first iteration of the CRS protocol. In contrast, learning curves shown by Shahaf et al had a very high initial value. Despite the erratic learning rates and the low initial value, we found that the cultures were trainable.

The learning experiments show that burst profiles are sensitive to changes in connectivity within the network. However, the direction of changes on evaluated electrodes was not uniform and could not be linked to changes in the SR relationship.

The increased rate of change of BPs during CRS, suggests that the whole network was involved in the training procedure. Since only one SR relationship is controlled, it may be that the rest of the network incorporates this change in a way that requires the least modifications. Conversely, it may also be that changes throughout the whole of network are driving the SR relationship toward its objective.

\section{ACKNOWLEDGMENT}

We thank Remy W. F. Wiertz for his work on the preparation and maintenance of cultures.

\section{REFERENCES}

Y. Jimbo, T. Tateno, and H. P. C. Robinson, "Simultaneous induction of pathway specific potetiation and depression in networks of cortical neurons.," Biophys J, vol. 76, pp. 670-678, 1999.

R. Madhavan, Z. C. Chao, and S. M. Potter, "Plasticity of recurring spatiotemporal activity patterns in cortical networks," Phys Biol, vol. 4, pp. 181-93, 2007.

E. Maeda, Y. Kuroda, H. P. Robinson, and A. Kawana, "Modification of parallel activity elicited by propagating bursts in developing networks of rat cortical neurones," Eur J Neurosci, vol. 10, pp. 488-96, 1998. 
[4] T. Tateno and Y. Jimbo, "Activity-dependent enhancement in the reliability of correlated spike timings in cultured cortical neurons," Biol Cybern, vol. 80, pp. 45-55, 1999.

[5] D. A. Wagenaar, J. Pine, and S. M. Potter, "Searching for plasticity in dissociated cortical cultures on multi-electrode arrays," Journal of negative results in biomedicine, vol. 5, 2006.

[6] G. Shahaf and S. Marom, "Learning in networks of cortical neurons," J Neurosci, vol. 21, pp. 8782-8, 2001.

[7] J. Stegenga, J. Le Feber, E. Marani, and W. C. Rutten, "Analysis of cultured neuronal networks using intraburst firing characteristics," IEEE Trans Biomed Eng, vol. 55, pp. 1382-90, 2008.

[8] H. J. Romijn, F. van Huizen, and P. S. Wolters, "Towards an improved serum-free, chemically defined medium for long-term culturing of cerebral cortex tissue," Neurosci Biobehav Rev, vol. 8, pp. 301-34, 1984.

[9] D. A. Wagenaar, T. B. DeMarse, and S. M. Potter, "MeaBench: A toolset for multi-electrode data acquisition and online analysis," presented at 2nd international IEEE EMBS conference on neural engineering, Arlington, VA, 2005. 